\title{
La suspensión de la presencialidad escolar: un resumen de la evidencia disponible a nivel internacional respecto de su efectividad para contener la actual pandemia de COVID-19
}

The suspension of school attendance: a summary of the evidence available at the international level regarding its effectiveness in containing the current COVID-19 pandemic

Agustín Ciapponi ${ }^{\mathrm{a}, \mathrm{b}}$

\begin{abstract}
Resumen
Tomando como excusa el análisis crítico del reciente informe suscripto por profesionales del Instituto de Cálculo de la Facultad de Ciencias Exactas y Naturales (UBA-CONICET) respecto del impacto de la suspensión de la presencialidad escolar como medida de control de la pandemia de COVID-19, que generó gran debate en Argentina, el autor de este artículo resume la evidencia a nivel internacional proveniente de revisiones sistemáticas sobre la eficacia y seguridad de este tipo de intervenciones, deteniéndose en cuándo, cómo y durante cuánto tiempo es razonable aplicarla. Por último, reflexiona y esboza propuestas que ayuden a fortalecer la racionalidad en la toma de decisiones.
\end{abstract}

\begin{abstract}
Taking as an excuse the critical analysis of the recent report by professionals from the Instituto de Cálculo de la Facultad de Ciencias Exactas y Naturales (UBA-CONICET) regarding the impact of the suspension of school attendance as a measure to control the COVID-19 pandemic, which generated much debate in Argentina, the author of this article summarises the international evidence from systematic reviews on the efficacy and safety of this type of intervention, focusing on when, how and for how long it is reasonable to apply it. Finally, he reflects on and outlines proposals to help strengthen rationality in decision-making.
\end{abstract}

Palabras clave: Escolaridad, Infecciones por Coronavirus, Políticas Públicas de Salud, Medicina Basada en la Evidencia. Keywords: Educational Status, Coronavirus Infections, Public Health Policy, Evidence-Based Medicine.

Ciapponi A. La suspensión de la presencialidad escolar: un resumen de la evidencia disponible a nivel internacional respecto de su efectividad para contener la actual pandemia de COVID-19. Evid Actual Pract Ambul. 2021;24(2):e002131. Available from: https://dx.doi.org/10.51987/EVIDENCIA.V24I3. 6950.

\section{El contexto}

La pandemia de COVID-19 ha tenido un impacto sin precedentes en el funcionamiento de las escuelas y los sistemas educativos en general. Las interrupciones en el funcionamiento y en el acceso a las escuelas han planteado nuevos desafíos para la salud, la economía, la educación y la protección de los niños. Pocas intervenciones han suscitado más polémica en Argentina (y en buena parte del mundo), en torno del abordaje de la pandemia, que el de la presencialidad escolar. El tema está tan atravesado por posiciones ideológicas, en medio de una campaña electoral y un intenso abordaje periodístico y de redes sociales, que incluso llegó a la corte Suprema de la Nación.

Se han escuchado afirmaciones muy dispares, como: "no hay ninguna evidencia de que la presencialidad se asocie a mayor contagio", "la suspensión de la presencialidad es claramente una de las medidas más eficaces para detener la circulación", "los datos muestran objetivamente que la presencialidad no aumentó los contagios", "este estudio demuestra rotundamente el impacto de detener la presencialidad" o "lo que se afirma es pseudociencia y militancia". Entonces me pregunto ¿Es posible que existan miradas tan opuestas del mismo problema?

Si pudiéramos descontextualizar por un momento el tema, podría decirse que el cierre escolar es sólo una medida no farmacológica basada en la comunidad, que intenta reducir las probabilidades de contagio de grupos de individuos asistentes a ámbi- tos educativos cerrados ( $y$ de manera indirecta, a sus contactos), reemplazando la educación presencial por la educación a distancia durante el mínimo período que las condiciones epidemiológicas lo requieran.

En teoría, no deberían generarse pasiones tan viscerales como las que están sucediendo. Todo comienza a complejizarse al comprender las múltiples aristas de las consecuencias de poner en práctica este tipo de decisiones. Pero el verdadero problema comienza, como tantas veces en investigación, por partir de la pregunta incorrecta. La pregunta no es si el remplazo de la presencialidad por la virtualidad es buena o mala en sí misma, como algunos han planteado. La respuesta es bastante obvia. Nadie en circunstancias normales plantearía suspender completamente la presencialidad por el impacto negativo que conlleva en términos de aprendizaje, socialización, condiciones laborales, financieras, logísticas, emocionales de niños y adolescentes, padres y cuidadores. Además, en ámbitos de bajos recursos, la presencialidad puede facilitar el acceso de los niños a los alimentos, aunque también puede implementarse la distribución de alimentos en domicilio en condiciones de educación virtual. Por todo, especialmente por la inequidad resultante, desfavoreciendo a los alumnos con menor acceso a los entornos digitales de aprendizaje, menor acompañamiento familiar y mayor inseguridad alimentaria, entre otros factores, eliminar la presencialidad tiene indudables consecuencias negativas. Sin embargo, no se trata de un dilema entre

\footnotetext{
a Servicio de Medicina Familiar y Comunitaria, Hospital Italiano de Buenos Aires.

b Centro Cochrane Argentina, Instituto de Efectividad Clínica y Sanitaria. aciapponi@iecs.org.ar
} 
salud y educación sino de replantear la pregunta para abordar el problema desde un punto de vista más integral: ¿Cuál es la opción que más minimiza el daño en una circunstancia epidemiológica determinada?

Habiendo establecido lo que considero el mejor punto de partida posible, intentaré desarrollar una respuesta con honestidad intelectual y lo más libre de preconceptos que me sea posible.

\section{La evidencia local}

Comenzaré con el informe realizado por profesionales del Instituto de Cálculo de la Facultad de Ciencias Exactas y Naturales (UBA - CONICET), por tratarse de un estudio a nivel local y por ser el que disparó el debate a las nubes ${ }^{1}$. El reporte señala que, en un contexto de alta cantidad de casos, con una incidencia de casos confirmados de 475 semanales/100 mil habitantes en el Conurbano bonaerense y de 610/100 mil habitantes en la Ciudad Autónoma de Buenos Aires (CABA), diversas medidas de mitigación junto con la suspensión temporal de la educación presencial en el Conurbano a partir del decreto de necesidad y urgencia (DNU) presidencial 241/2021 se asociaron con una disminución más veloz y pronunciada de la cantidad de casos confirmados, en comparación con lo sucedido en la CABA y en el resto de la Provincia de Buenos Aires (PBA), donde se mantuvieron las clases presenciales. El estudio se apoya en la gráfica de los casos confirmados en la población general, por fecha de inicio de síntomas (FIS), o por fecha de apertura para casos con FIS ausente, con datos de la base del Ministerio de Salud de Nación actualizados al 08/05/2021 hasta 28/04/2021 (ver Figura 1, arriba).

Las primeras restricciones a la nocturnidad anunciadas el 07/04 se asociaron a un cambio de tendencia al día 10/04, con una pendiente levemente negativa en el Conurbano y levemente positiva en CABA y el interior de la PBA. Las segundas restricciones anunciadas el 14/04 fueron seguidas de un cambio de tendencia al día 20/04, con una pendiente negativa muy similar en las tres regiones. Luego del cierre de la educación presencial el 19/04, en el Conurbano se observa una mayor negativización de la pendiente a partir del 22/04. La disminución de los casos entre el $22 / 04$ y el $27 / 04$ fue de $22,0 \%$ en el Conurbano, de $13,2 \%$ en CABA y de $11,4 \%$ en interior de la PBA. Estos cambios de pendiente se observaron entre los tres y los seis días posteriores a los anuncios de las restricciones, en forma coincidente con la duración más frecuente del período de incubación del SARS-COV-2. Las diferencias de pendiente entre regiones fueron mucho más marcadas en la franja etaria de 5 a 11 años (ver Figura 1, abajo).

El diseño de este estudio corresponde al de serie de tiempo interrumpida (STI). Este tipo de abordaje quasi-experimental es uno de los más poderosos para evaluar políticas de salud pública, pero debe cumplir con criterios de calidad metodológica para que la certeza de la evidencia no se vea afectada. Utilicé la herramienta del grupo Cochrane denominada Práctica Efectiva y Organización de los Cuidados (en inglés, Effective Practice and Organisation of Care: EPOC) que consta de siete ítems ${ }^{2}$, para valorar el riesgo de sesgo del estudio mencionado:

1. La intervención se sumó a otras medidas previas, separadas en el tiempo, lo que suele ocurrir en este tipo de diseño de investigación. En este caso, hubo otras intervenciones concurrentes, como las restricciones en las franjas horarias nocturnas, en el transporte público, en la circulación de áreas comerciales, en la gastronomía, en las actividades culturales y recreativas o una eventual autorregulación de los comportamientos sociales. Sin embargo, la inspección visual muestra que la pendiente más marcada de descenso se produjo tras tres días de suspensión de la presencialidad en el Conurbano. Incluso de haber sido parcialmente influyentes, estas otras intervenciones concurrentes serían insuficientes para explicar los posibles efectos diferenciales por región o grupo etario: bajo riesgo de un efecto diferencial espurio. Sin embargo, debemos ser cautos a la hora de interpretar la potencial influencia de otros eventos o intervenciones concurrentes como las mencionadas durante el período del estudio, que pudieron haber sido diferentes en distintas áreas geográficas: riesgo incierto.

2. La forma ("shape") del efecto de la intervención se basa en una explicación racional proporcionada por los autores, esperando cambios de pendiente entre los tres y los seis días posteriores a los anuncios de restricciones, según el período de incubación del SARS-COV-2: bajo riesgo.

3. Es poco probable que la intervención (suspensión de la presencialidad escolar en Conurbano) haya afectado por sí misma la recolección de datos antes y después de la intervención en las diferentes regiones. Sin embargo, dado que los niños permanecieron escolarizados en forma presencial en CABA, eso pudo haber influido en que en esa localidad hubiera un sesgo hacia una mayor detección de casos infantiles que en las jurisdicciones del conurbano bonaerense, donde los niños tuvieron un menor acceso a ser testeados, por no estar concurriendo a los establecimientos escolares. Dado que la infección tiene altas probabilidades de cursar en forma asintomática a dicha edad, esa diferencia en las pendientes de los casos incidentes podría ser explicada, en parte, a partir de la diferente "intensidad" en el testeo lo cual implicaría riesgo de sesgo. Este riesgo habría sido menor si la investigación hubiese considerado como medida de efecto a algún resultado más objetivo o "duro" como es la mortalidad, la incidencia de nuevos casos de personas con ventilación mecánica o aún, indicadores indirectos como la proporción de ocupación de camas hospitalarias (ver un ejemplo local en la figura 2). Por estas razones consideramos que este sesgo de riesgo incierto

4. Los resultados primarios corresponden a la hipótesis o pregunta primaria de los autores. Si bien las intervenciones fueron de público conocimiento (es decir, que no hubo cegamiento), las variables de resultado, obtenidas de datos oficiales durante el estudio, eran lo suficientemente objetivas como para haber sido influenciadas por la falta de cegamiento: bajo riesgo.

5. Es poco probable que las medidas de resultado faltantes sesguen los cambios de pendiente, asumiendo que la proporción de datos faltantes fue similar en los períodos anterior y posterior a la intervención. Si bien la pérdida de datos puede ser diferente entre CABA y en la PBA (sugerida por una mayor tasa de positividad de testeos en la provincia), los cambios de tendencia se establecen en relación a la tendencia previa de cada región. Aun asumiendo un subregistro de la PBA en relación a CABA, dentro del mismo sistema de registro pudo observarse un efecto diferencial entre Conurbano y el interior de la provincia: bajo riesgo de un efecto diferencial espurio.

6. No hay evidencia de que los resultados se hayan informado de manera selectiva o que se hayan omitido resultados importantes, aunque al carecerse de un protocolo previo no podríamos estar seguros: riesgo incierto.

7. No hay evidencia de otros riesgos de sesgo, como la estacionalidad, durante el breve período de estudio: bajo riesgo.

Por otro lado, el estudio presenta como limitación el escaso número de puntos de la serie (a menor cantidad de puntos, menor esta- 
bilidad de las tendencias) y la falta de un análisis estadístico formal para serie de tiempo interrumpidas, como modelos ARIMA o de regresión de series de tiempo ajustados por la correlación serial. Por lo tanto, no puede afirmarse si las diferencias observadas fueron estadísticamente significativas. Por otro lado, debe destacarse que el efecto diferencial observado entre regiones y grupos etarios, podría estar diluido si se considera que la presencialidad en CABA y en el interior de la PBA fue muy reducida durante la semana del 19/04 al 23/04 (contaminación de intervenciones) y también por la permanente circulación de personas (y generación de contactos) entre el Conurbano y CABA, ya que la mezcla de dos poblaciones con incidencias diferentes, así como la contaminación, siempre tiende a su homogeneización y a diluir las diferencias: bajo riesgo de un efecto diferencial espurio. Además en un contexto de alta incidencia viral y con una mayor circulación comunitaria de variantes más contagiosas como B.1.1.7 (Reino Unido), P1 (Manaos), las diferencias en términos absolutos podrían magnificarse ${ }^{3}$.

Se argumentó que el estudio presentaba conclusiones inválidas por las diferencias de cómo se miden los casos positivos en cada distrito, destacándose que en los últimos tres meses, CABA realizó al menos cinco veces más pruebas por millón de habitantes que el Conurbano por lo que es esperable que detecte más casos. Esto también reflejado en la positividad de las pruebas, que en la PBA adquiere valores que casi duplican a los de la $\mathrm{CABA}^{4}$. Si bien puede haber habido una menor tasa de testeo en los niños y familias que no están concurriendo a la escuela, es probable que la magnitud del cambio desde la intervención no haya sido importante. Por lo tanto, no coincido con esta crítica ya que omite decir que los cambios de tendencia de una serie se establecen sobre su propio comportamiento previo, luego de la "interrupción" por la intervención en estudio.

Aunque el enfoque GRADE ${ }^{5}$ para valorar la certeza de la evidencia está pensado para "cuerpos de evidencia", haremos el ejercicio para este único estudio que aborda la cuestión en Argentina. Lo tradicional es comenzar calificando los estudios no aleatorizados como éste, de "baja certeza" 6 , aunque las STI con múltiples períodos y mediciones durante cada período sin otras limitaciones podrían constituir evidencia de "certeza moderada" 7,8 . Digamos que en este caso la certeza de la evidencia podría considerarse baja (o muy baja si se jerarquiza una posible imprecisión por la falta de análisis formal, aunque contrarrestada en parte por la subestimación del efecto en un contexto creciente de cepas virales más transmisibles).

Considero que no puede asegurarse que este estudio constituya una evidencia suficiente de que la suspensión de la presencialidad escolar tiene un impacto causal respecto de la disminución de los contagios. Sus conclusiones podrían haber sido más moderadas, especialmente dado que la principal medida de resultado fue la de casos incidentes, muy sensibles a la intensidad de los testeos. Sin embargo, no adhiero a las críticas de que sus conclusiones deberían ser consideradas inválidas, sino de baja certeza. Las mismas merecen ser evaluadas en futuros estudios, por ejemplo, una STI controlada también por resultados que no sean sensibles a las intervenciones de limitación de la circulación viral.

Sería interesante la continuación de la serie (si se adoptaran conductas diferenciadas a nivel geográfico en el futuro) pero prestando atención a la tasa de testeos antes y después de las intervenciones ("interrupciones de la serie"). En esta oportunidad sería conveniente análisis estadísticos formales para STI para establecer la significancia estadística de los cambios de pendiente.

\section{¿Qué es lo ha venido ocurriendo en el mundo en cuan- to al cierre de establecimientos educativos?}

Para combatir la propagación del SARS-CoV-2 y los efectos de la COVID-19, los países de todo el mundo vienen adoptado diversas medidas de salud pública. En muchos de ellos, el cierre de las escuelas fue una de las primeras respuestas. A mediados de abril de 2020, 192 países habían cerrado en algún momento sus centros escolares, lo que incluyó a más del $90 \%$ de la población estudiantil mundial (casi 1.600 millones de estudiantes) ${ }^{9}$ Entre otras consecuencias negativas ${ }^{10}$, los cierres de escuelas tienen posibles implicancias psicosociales y económicas, como en los resultados educativos, la salud física y mental ${ }^{9,11}$. Debido a estos posibles efectos negativos del cierre de los establecimientos escolares o a la evolución favorable de las condiciones epidemiológicas, muchos países han realizado reaperturas, y a la fecha hay 24 estados (involucrando a casi 211 millones, o 12\% de los estudiantes del mundo) que mantienen cerradas las escuelas a nivel nacional (ver Figura 3).

Dentro del anteproyecto de ley de emergencia COVID, el Poder Ejecutivo plantea la suspensión de clases presenciales en los lugares de alarma epidemiológica. Sobre esto, define .alarmaçuando hay más de 500 casos cada 100.000 habitantes y más de $80 \%$ de ocupación de camas de terapia intensiva. Según este proyecto de ley, los parámetros propuestos para definir la situación epidemiológica son los siguientes: bajo riesgo: entre 50 y 150 casos cada 100.000 habitantes; mediano riesgo: entre 150 y 250 contagios; alto riesgo: entre 250 y 500 casos; y alarma epidemiológica: más de 500 casos positivos y $80 \%$ de ocupación de las unidades de terapia intensiva. En situaciones de alto riesgo epidemiológico, el Gobierno podrá disponer de medidas en los conglomerados urbanos que tengan más de 300.000 habitantes, que son lugares en donde se presenta una mayor complejidad en la prevención de la circulación del virus. Las medidas que se adopten tendrán un plazo máximo de 21 días y se evaluarán sus resultados para determinar si mejoraron las curvas epidemiológicas. Por su parte, las autoridades locales podrán disponer medidas focalizadas en lugares de menos de 40.000 habitantes y en situaciones de mediano riesgo, más allá de las restricciones vigentes en todo el país ${ }^{12}$.

\section{La evidencia internacional}

Es de suma importancia analizar la evidencia internacional acerca de la transmisión del SARS-CoV-2 en las escuelas para respaldar la toma de decisiones sobre el cierre o reapertura de las instalaciones educativas con medidas de prevención y control efectivas durante la pandemia. A tal fin hice una revisión rápida en la base de datos Epistemonikos de revisiones sistemáticas para la toma de decisiones en salud ${ }^{13}$ y en las listas de referencias de las revisiones sistemáticas incluidas.

Los cierres de escuelas se han venido implementado basados en la respuesta a brotes anteriores de enfermedades transmisibles, en particular la influenza, pues los niños pueden jugar un papel clave en la transmisión de muchas enfermedades virales ${ }^{14}$ Los estudios de simulación han sugerido que el cierre escolar puede ser una medida de control útil durante una pandemia de influenza, en particular para reducir la demanda pico en los servicios de salud. Sin embargo, para las epidemias de influenza, no hay evidencia concluyente sobre los cierres escolares. Una revisión sistemática ${ }^{15}$ encontró que los cierres de escuelas redujeron un $30 \%$ el pico de casos, retrasándolo 11 días, mientras que otra revisión sugirió que la reducción en la incidencia y transmisión de la influenza varió ampliamente entre el $1 \%$ y el $50 \%{ }^{16}$. A diferencia de la influenza, en la que las escuelas son importantes contribuyentes a la transmisión, los estudios escolares sobre el SARS-CoV-2 son más limitados debido al cierre reactivo de las escuelas. 
Figura 1. Efecto de las restricciones nocturnas y la suspensión de la escolaridad presencial (aplicada al Conurbano bonaerense) sobre los nuevos casos en la población total y en la franja etaria de 5 a 11 años. Fuente: Modificado de la Figura 3 Castro $\mathrm{R}$ et al, agregando el día de anuncio del 7/4/2021 de las restricciones nocturnas a las 23.00 horas (1), el del 14/4/2021 a las 20.00 horas (2) y el del el 19/04/2021 del paso a la escolaridad virtual en el Conurbano bonaerense (3).

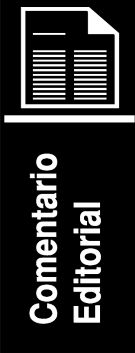

\section{Casos relativos al 22/04 para todos los grupos etarios}
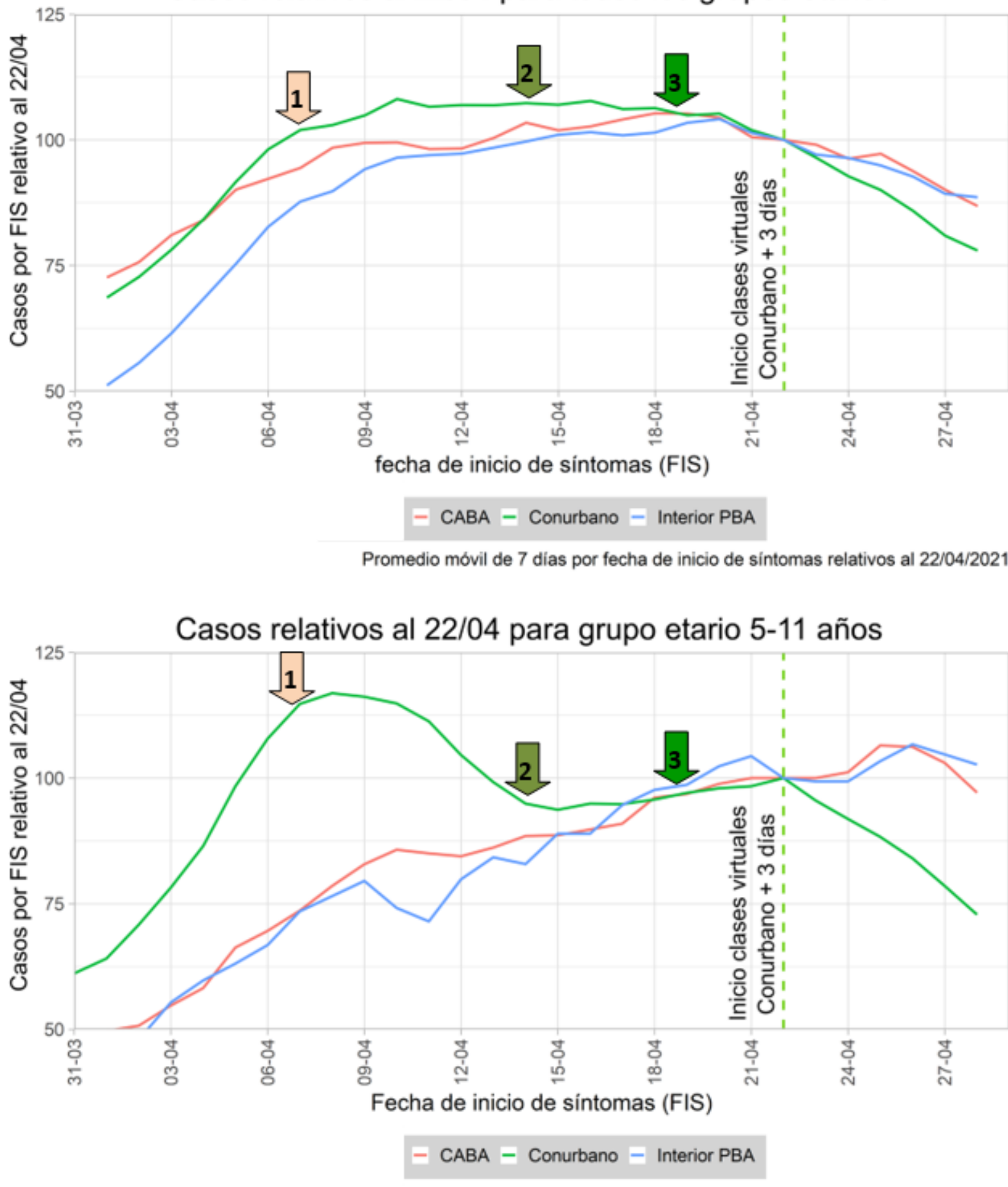
Figura 2. Evolución de la cantidad de camas ocupadas en un Hospital Universitario Privado de la Ciudad Autónoma de Buenos Aires, Argentina. Fuente: Cortesía de Hernán Michelángelo. Extracto de un Informe de la Gestión de la Internación vinculado a la Pandemia de COVID-19 (09/06/2021). Agradecemos a la Dirección del Hospital Italiano de Buenos Aires, el permiso para su reproducción.

\section{CAMAS HOSPITALARIAS OCUPADAS Y PROYECTADAS}



Una revisión sistemática sintetizó la evidencia sobre el uso, la efectividad y la costo-efectividad del cierre de escuelas y diversas prácticas de distanciamiento social para reducir las tasas de infección y la transmisión durante brotes de coronavirus (SARSCoV-1, MERS-CoV, SARS-CoV-2) en las escuelas ${ }^{17}$. Sin embargo, la mayoría fueron estudios de modelos, que aún no disponían de evidencia empírica sobre COVID-19 y, debido a la heterogeneidad sustancial de los estudios incluidos, no se realizó un meta-análisis. Un estudio de modelado, ampliamente citado sobre COVID-19, también incluido en esta revisión, predijo que el cierre de escuelas y universidades, implementado junto con una variedad de otras intervenciones, podría prevenir entre el $2 \%$ y el $4 \%$ de las muertes ${ }^{18}$. La evidencia basada en estudios de modelos más recientes encontró que la efectividad de los cierres combinados de escuelas y universidades es mucho mayor que el inicialmente predicho, estimándose una reducción del $21 \%$ al $55 \%$ en el número de reproducción $\mathrm{R}^{17}$.

Una revisión sistemática con meta-análisis investigó específicamente el alcance de la transmisión del SARS-CoV-2 en las escuelas. Incluyó 11 estudios realizados en países de altos ingre$\operatorname{sos}^{19}$. Cinco estudios de cohorte reportaron que, en total, 22 casos índice de estudiantes y 21 del personal escolar expusieron a 3.345 contactos con 18 transmisiones (tasa combinada de ataque de infección estimada de $0,08 \%$; intervalo de confianza [IC] del $95 \% 0,00$ a 0,86). Las tasas para estudiantes y personal escolar fueron $0,15 \%$ y $0,70 \%$, respectivamente. Seis estudios transversales informaron una tasa combinada de positividad para el SARS-CoV-2 de $8,00 \%$ (IC $95 \% 2,17$ a 16,95). Fue de $8,74 \%$ entre los estudiantes y de $13,68 \%$ en el personal de la escuela Los brotes en racimo tuvieron poca frecuencia relativa (solo uno de cinco países de un estudio de cohortes). En general, la calidad de los estudios se consideró deficiente, lo que limitó la confianza en los resultados. Los autores concluyeron que existe limitada evidencia de alta calidad disponible como para cuantificar el alcance de la transmisión del SARS-CoV-2 en las escuelas o para compararla con la transmisión comunitaria, aunque en general parecería ser baja y menor en los estudiantes que en el personal escolar ${ }^{19}$

Una revisión sistemática incluyó diez estudios, con datos de 146 países, que evaluaron el cierre de escuelas y uno examinó, además, las reaperturas. Tres estudios, incluidos los dos con menor riesgo de sesgo, no informaron impacto alguno del cierre de escuelas en la transmisión del SARS-CoV-2, mientras que los otros siete informaron efectos protectores. Los tamaños del efecto variaron desde ninguna asociación hasta reducciones sustanciales e importantes en la transmisión comunitaria. Los estudios presentaban riesgo de confusión y colinealidad debido a otras intervenciones no farmacológicas implementadas casi simultáneamente al cierre de escuelas. Sus resultados sobre los cierres de escuelas van de ineficaces a muy efectivos 20 .

Una revisión sistemática incluyó 22 estudios y consideró que la literatura evaluada proporcionó pruebas suficientes de que los niños pueden ser infectados por el SARS-CoV-2 y transmitirlo en la comunidad, el hogar y en la escuela. La transmisión por niños se documentó con mayor frecuencia en entornos domésticos, mientras que los ejemplos de niños como "casos índices" en entornos escolares fueron más raros. Los estudios incluidos sugi- 
Figura 3. Seguimiento mundial del cierre de escuelas causados por el COVID-19. Fuente: https://es.unesco.org/covid19/educati onresponse. Nota: En la web de UNESCO es posible visualizar la evolución de los cierres a lo largo del tiempo

Seguimiento mundial de los cierres de escuelas causados por el COVID-19



No data
Visualizar la evolución en el tiempo

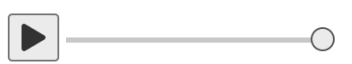

$11 / 06 / 2021$

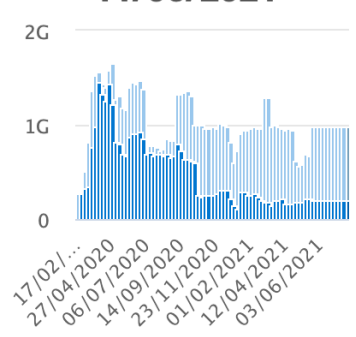

$198,613,483$ estudiantes afectados

$11.3 \%$ del total de alumnos matriculados 21 cierres a nivel nacional

(CUNESCO. UN Disclaimers rieron que el cierre de escuelas puede ayudar a reducir la transmisión del SARS-CoV-2, pero que debe considerarse el impacto social, económico, y educativo de cierres prolongados de los establecimientos educativos ${ }^{21}$.

Una revisión sistemática incluyó tres estudios epidemiológicos, y cinco estudios de modelado que utilizan datos reales sobre cierres escolares ocurridos generalmente luego del pico epidémico. El cierre de las escuelas casi redujo la transmisión del COVID-19 cuando la curva de casos comenzaba a descender24. La demora en el cierre de escuelas y las combinaciones con otras medidas de control y gestión dificultaron sacar conclusiones consistentes sobre la efectividad del cierre de escuelas en COVID-1922.

Una revisión sistemática incluyó 36 evaluaciones económicas completas, publicadas entre 1998 y 2020, sobre las medidas de salud pública aplicadas durante las pandemias de influenza y COVID-19. La mayoría de los estudios se llevaron a cabo en países de altos ingresos y solo algunos de los estudios fueron sobre intervenciones no farmacológicas. La mayoría de las intervenciones fueron costo-efectivas en varios escenarios incluso desde la perspectiva social, mientras que el cierre de escuelas sólo lo fue en un escenario de .alta tasa de letalidad". El nivel de infectividad y gravedad de la pandemia fueron los factores clave de la costo-efectividad de las intervenciones farmacológicas y no farmacológicas ${ }^{23}$.

Un estudio evaluó más de 45 tipos diferentes de medidas preventivas destinadas a la reducción de la circulación del virus, aplicadas en territorios de más de 100 países. Concluyó que el cierre de instituciones educativas es la segunda medida más efectiva para reducir la circulación viral (luego de la cancelación de las reuniones sociales). Estos autores también concluyeron que ninguna medida es suficiente por sí misma, ubicando al cierre de escuelas como una de las principales intervenciones a ser combinada con otras acciones. Los autores resaltan que la creciente evidencia provista por los estudios más recientes ha revertido la presunción inicial de que el cierre de escuelas no impacta sustancialmente en la transmisión comunitaria de $\operatorname{casos}^{24}$

Un estudio evaluó 13 intervenciones no farmacológicas sobre la transmisión de COVID-19 en 130, encontrando una fuerte evidencia de una asociación en dos de ellas (el cierre escolar y las restricciones de movimiento interno) y una menor tasa de reproducción (Rt) ${ }^{25}$. Asimismo, un estudio evaluó el efecto de intervenciones no farmacológicas sobre la transmisión de COVID-19 en 41 países durante la primera ola de la pandemia y concluyó que cerrar todas las instituciones educativas, limitar las reuniones a 10 personas o menos, y cerrar negocios cara a cara redujo considerablemente la transmisión ${ }^{26}$.

Los argumentos taxativos de que las escuelas no contribuyen a la transmisión comunitaria y de que el riesgo general de COVID19 para los niños es insignificante, han generado que las medidas de mitigación de contagios en las escuelas hayan recibido escasa prioridad. Sin embargo, la evidencia citada para estos argumentos tiene serias limitaciones ${ }^{27,28}$. Podría resumirse, sobre los cierres escolares, que evidencia de baja calidad proveniente en su mayoría de países de altos ingresos, sugiere que la transmisión sería baja en condiciones escolares apropiadas. Sin embargo, las diferencias en cuanto a la efectividad y los efectos perjudiciales, ya sean reales o espurias considerando la baja certeza del cuerpo de la evidencia, hacen difícil arribar a conclusiones sólidas. Para implementar de manera eficiente el cierre de escuelas, previamente debiéramos responder con más precisión la siguiente pregunta.

\section{Cierre vs. apertura escolar ¿Cuándo, cómo y durante cuánto tiempo?}

Tanto los modelos matemáticos como los datos del mundo real sugieren que la apertura de todas las escuelas sin medidas de mitigación sólidas probablemente conduzcan a que la Rt se eleve por encima de 1 en casi todos los escenarios (es decir, crecimiento exponencial de los contagios) ${ }^{29}$. Las mitigaciones de varios 
niveles pueden reducir sustancialmente el riesgo de transmisión dentro de las escuelas y los hogares ${ }^{30}$.

Sin embargo, no puede obviarse que existen barreras para cumplir las recomendaciones de salud pública para evitar el contacto social durante el cierre escolar. Una revisión sistemática incluyó 19 estudios y encontró que las actividades y los contactos sociales parecían disminuir durante los cierres, pero el contacto seguía siendo común, pues los niños salían de sus casas o eran cuidados por personas ajenas al hogar. Hubo alguna evidencia de que la mayor edad de los niños y el desacuerdo de los padres con el cierre eran predictivos de que los niños salieran de sus casas. El acuerdo de los padres con el cierre fue en general alto, pero algunos padres no estaban de acuerdo debido al bajo riesgo percibido de infección, problemas prácticos relacionados con el cuidado de los niños y el impacto financiero de las medidas en el hogar ${ }^{31}$.

Por otro lado, existe evidencia de que los padres pueden ser el caso índice contagiante de los niños infectados por COVID19 mientras los niños permanecen confinados en el hogar tras el cierre de las escuelas. Una revisión sistemática documentó que la prevalencia de padres como caso índice fue del $54 \%$ (IC $95 \% 29$ a 79 ) y en el $70 \%$ de los casos, estos padres eran sintomáticos al momento del contagio. Por lo tanto, desde una perspectiva de salud pública, la detección temprana de estos padres es crucial para una reapertura segura ${ }^{32}$.

Una revisión sistemática identificó estudios sobre la implementación de la educación infantil durante la pandemia de COVID19. Se documentó que la mayoría de los niños experimentaron el aprendizaje a distancia para reducir la propagación del brote, especialmente cuando no se puede llevar a cabo el aprendizaje cara a cara. Los padres y maestros tienen un papel importante en el apoyo a los niños para mejorar su desarrollo cognitivo y social. Sin embargo, la implementación del aprendizaje en el hogar causa algunas dificultades en relación con la autorregulación de los niños muy pequeños, la disposición para utilizar la tecnología digital y los materiales de aprendizaje, las creencias y actitudes de los padres sobre el aprendizaje remoto en comparación con el aprendizaje tradicional y los requisitos de tiempo y conocimiento que exige el acompañar a los niños. Por lo tanto, concluyó que es importante tener una buena comunicación entre padres y maestros para apoyar este tipo de aprendizaje ${ }^{33}$.

Una revisión Cochrane de alcance (scoping review) identificó las medidas escolares para contener la COVID-19 como base de una futura revisión sobre la efectividad de estas medidas que ayudará a documentar las guías de la Organización Mundial de la Salud (OMS) ${ }^{34}$. Fueron identificados 42 estudios que evaluaron medidas en más de un ámbito de enseñanza primaria y secundaria (31 estudios de modelización matemática, dos diseños experimentales y nueve estudios observacionales), la mayoría de ellos realizados en Europa y América del Norte y del Sur. Los estudios evaluaron tres tipos de medidas generales que deberían ser consideradas por los decisores:

1. Medidas organizativas para reducir la transmisión del SARS-CoV-2 (36 estudios) como las mascarillas, las normas de distanciamiento físico y las medidas para reducir las ocasiones de contacto (por ejemplo, el escalonamiento de las horas de llegada, recreo y salida).

2. Medidas estructurales 0 ambientales para reducir la transmisión del SARS-CoV-2 (11 estudios) como dividir los patios de las escuelas o mejorar la circulación del aire.

3. Medidas de vigilancia y respuesta para detectar las infecciones por SARS-CoV-2 (19 estudios) que incluyeron las pruebas, el rastreo y la detección de los síntomas y el aislamiento de los casos confirmados o la cuarentena de los casos sospechosos.
Una revisión rápida describió las principales medidas previstas en Europa para el año académico 2020-2021 e incluyó 23 documentos oficiales (Francia, Luxemburgo, Malta, Irlanda, Italia, Portugal, Reino Unido, España y San Marino) y siete artículos académicos. El cierre de escuelas fue considerado generalmente como el último recurso para el control de la COVID-19. Las recomendaciones sobre el cierre escolar coincidían en su mayoría en que esta medida debía ser el último recurso para el control de la pandemia, no así en los criterios para el regreso a la escuela de los estudiantes que dieron positivo y la flexibilidad entre la asistencia a la escuela y la educación remota para los niños de alto riesgo ${ }^{35}$.

La Organización Panamericana de la Salud (OPS) publicó una guía que analizó las consecuencias sanitarias, sociales y económicas de las medidas tomadas para el control de la pandemia relacionadas con el cierre de escuelas, así como los elementos que deben tenerse en cuenta a la hora de reabrirlas, con un enfoque en la niñez y la adolescencia en situaciones de vulnerabilidad. Proporciona a equipos técnicos de los Ministerios de Salud y Educación y tomadores de decisiones locales, las consideraciones para asegurar que los planes para reabrir las escuelas incluyan acciones especialmente diseñadas para beneficiar a los niños y adolescentes con mayor riesgo educativo y sanitario. Estas sugerencias se focalizan en el apoyo para la salud y el bienestar de los estudiantes y docentes, en hacer participar a las familias, las comunidades y los interesados directos locales en los planes de reapertura de las escuelas, en procurar que los niños, niñas y adolescentes con alto riesgo de resultados negativos en materia de salud y educación regresen a la escuela cuanto antes, en garantizar que haya agua, saneamiento e higiene al volver a las escuelas, y en facilitar el acceso al aprendizaje en línea ${ }^{36}$. Por su parte, la OMS estableció una lista de verificación para apoyar a las escuelas en la reapertura y en la preparación ante rebrotes de COVID-19 y el resurgimiento de crisis similares de salud públicas $^{37}$, en la que se detallan las acciones esenciales que se deberían tener en cuenta, en una articulación multinivel, en las distintas fases de reapertura y preparación para el recrudecimiento de las tasas de incidencia de esta enfermedad.

En Argentina, el Consejo Federal de Educación aprobó en Julio de 2020 el "Protocolo marco y lineamientos federales para el retorno a clases presenciales en la educación obligatoria y en los institutos superiores", en el que se establecen las condiciones para la reapertura de clases presenciales y se establece un protocolo de referencia con los cuidados mínimos a tener en cuenta, que debe ser cumplido y adaptado por cada jurisdicción ${ }^{38}$. Este documento enuncia una serie de acciones previas a la reapertura de la presencialidad y acciones durante la apertura de los establecimientos escolares, además de los lineamientos pedagógicos generales. Entre las medidas de mitigación previstas para una presencialidad segura se enuncian a) las pautas de actuación ante la aparición de caso/s sospechoso/s o confirmado/s en el ámbito escolar, estableciendo diferentes escenarios posibles de implementación de la escolaridad presencial (estrategia de grupos de alumnos por aula versus estrategia de "burbujas", esta última referida como la posibilidad de mantener grupos, cuyos integrantes tendrán el distanciamiento físico adecuado entre sí, diferenciados en toda la instancia educativa, o sea, dentro de aula y los espacios comunes del establecimiento), entre las que se cuentan el aislamiento preventivo de los contactos y la indicación del testeo para detección de COVID-19; b) las pautas de distanciamiento social; c) las medidas de higiene personal (de manos y respiratoria, destacándose la recomedación del uso de tapabocas durante todo el tiempo que dure la permanencia en la escuela); d) los lineamientos para la identificación y seguimiento del personal o alumnos/as en riesgo, detallando los grupos de riesgo exentos de concurrir a actividades presenciales; e) y las 
medidas de actuación para casos sospechosos y contactos estrechos, entre las que se detallan las actividades de identificación temprana de potenciales casos, el impedimento del ingreso a las instituciones educativas de alumnos o personal con síntomas de sospecha de COVID-19, y el aislamiento y la notificación de los casos sospechosos a la autoridad sanitaria. Además se establecen recomendaciones para el transporte público y escolar, y para la higiene, seguridad y acondicionamiento de la infraestructura de los establecimientos educativos, incluyendo la descripción de las técnicas de limpieza y desinfección.

Por otro lado, el plan estratégico de vacunación de la nación establece al personal docente y no docente dentro de los grupos priorizados por riesgo de exposición y función estratégica, medida tendiente a garantizar el adecuado cuidado de la salud de los trabajadores de la educación ${ }^{39}$.

Un reciente estudio basado en datos de una encuesta masiva en línea en los EE.UU. (COVID-19 Symptom Survey ${ }^{40}$ ) indicó que los convivientes de los niños que asisten a una jornada escolar completa y semicompleta, presentan un aumento de casos. Aun cuando la transmisión dentro del aula pueda ser infrecuente, las actividades sociales asociadas con la escolaridad presencial (como el traslado de los estudiantes, las interacciones entre los docentes y el comportamiento social cuando las escuelas están abiertas) pueden conducir a incrementos en los niveles de transmisión comunitaria. Las medidas de mitigación basadas en la escuela estuvieron asociadas con reducciones significativas en el riesgo, particularmente el rastreo de síntomas diarios, el uso de barbijos, la implementación de burbujas, la reducción del alumnado, la ventilación de las aulas y el cierre de actividades extracurriculares. De hecho, cuando se asocian siete o más medidas de mitigación, ya no se observa una relación significativa de la escolaridad presencial con el aumento de los casos ${ }^{41}$.

Otra investigación realizada en ese país mediante el seguimiento activo de casos, documentó que la transmisión secundaria de COVID-19 en las escuelas en las que se implementó la educación presencial acompañada del uso asiduo de máscaras faciales, distancia social e higiene de manos fue muy limitada ${ }^{42}$.

Es previsible que, como ocurre en otros países, la implementación de las medidas de mitigación en Argentina sea heterogénea. Lamentablemente, no existe un repositorio de acceso público sobre los diversos protocolos institucionales aprobados ni estudios que describan el grado de adopción en la práctica de las diversas medidas propuestas por el Consejo Federal de Educación. Sin embargo, según datos recolectados por la Plataforma Cuidar Escuelas, al 6 de Abril del 2021, en una muestra analizada de 5.926 establecimientos activos, a los que asisten un total de 1.429 .190 estudiantes matriculados y 214.850 docentes y no docentes, se informó que la incidencia de COVID-19 en el sistema educativo fue baja: sobre el total de estudiantes matriculados en 2021 representaba el $0,12 \%$ y el $0,79 \%$ del cuerpo de directivos, docentes y auxiliares ${ }^{43}$.

\section{Reflexiones finales}

La suspensión de la presencialidad escolar ha sido y sigue siendo una medida utilizada con frecuencia por los países frente a altos niveles de circulación viral. La evidencia apoya la idea de que la educación presencial conlleva un mayor riesgo de transmisión de COVID-19, particularmente en contextos de alta circulación viral y cuando no se aplican medidas de mitigación en la escuela y alrededores, o si no se garantiza un traslado seguro de estudiantes, docentes y cuidadores. En estos casos la interrupción de la presencialidad escolar puede contribuir significativamente a disminuir contagios, y secundariamente, internaciones y fallecimientos, y es así que muchos estudios la vindican como una de las medidas no farmacológicas de mayor impacto.
Sin embargo, es más difícil estimar el impacto de las medidas de mitigación implementadas en los distintos contextos de nuestro país en la transmisión del COVID-19, y por lo tanto, conocer la relación de costo-beneficio de un cierre de los establecimientos escolares en comparación con la evaluación y el refuerzo de los protocolos en los casos en que se detecte una implementación insuficiente, ecuación en la que además debiera incorporarse el grado de implementación de la virtualidad educativa como reemplazo de la escolaridad presencial.

La pandemia está lejos de haber terminado. El cierre temporario de los establecimientos educativos es una herramienta para la reducción de contagios, a la que posiblemente se deba recurrir en más de una oportunidad, aplicándola con eficiencia. Más aun considerando las cuestiones estacionales y las nuevas variantes virales más transmisibles en circulación. Considero que no sólo debiéramos inmunizarnos contra el COVID-19, sino también contra las innumerables influencias coyunturales que puedan afectar el manejo racional de la presencialidad escolar. Por eso apoyo la idea de alcanzar un amplio consenso que active o desactive el cierre de escuelas según la particular circunstancia epidemiológica de áreas geográficas ampliamente interconectados como lo es el Área Metropolitana de Buenos Aires. Podrá discutirse qué indicadores epidemiológicos deberían tenerse en cuenta y sus valores umbrales a partir del cual las medidas se activarían, pero en cualquier caso, aislar estas decisiones de posiciones ideológicas, vaivenes electorales o encuestas de opinión. Algunos países, como por ej., Alemania, proponen establecer límites a la continuidad de la presencialidad en contextos epidemiológicos de alta incidencia de infección por SARS-CoV-2. En el caso de ese país, un proyecto de ley determina como indicador la cantidad de casos semanales detectados cada 100 mil habitantes, previendo un umbral de 165 cada 100 mil habitantes para la suspensión de la educación presencial ${ }^{44}$.

Estas consideraciones por supuesto no desconocen que la educación presencial es indiscutiblemente ventajosa frente a la educación en modalidad virtual exclusiva, la cual presenta a su vez dificultades y a menudo reproduce, genera y amplifica desigualdades socioeconómicas y de género. Por lo tanto, no debe extenderse indefinidamente en el tiempo y, en simultáneo, deben promoverse acciones para facilitar la virtualidad y compensar el apoyo alimentario que brindan las escuelas para reducir, al menos en parte, las inequidades existentes.

Por otro lado, si bien regresar a la escuela lo antes posible es imperativo para la educación, el desarrollo social y el bienestar mental y físico de los niños, es necesario garantizar que las escuelas sean más seguras para los estudiantes y el personal. Existe evidencia científica de que hay 20 veces más posibilidades de infectarse en interiores que en exteriores. Todos los casos de superpropagación se advierten en los sitios con mala ventilación, mientras que no se ha documentado la superpropagación por gotas o superficies contaminadas (el contagio por superficies se estima en sólo uno cada 10 mil contagios) ${ }^{45}$. La transmisión a partir de personas asintomáticas, que no tosen ni estornudan, pero contagian por aerosoles puede llegar hasta $59 \%$ (35\% individuos presintomáticos y $24 \%$ individuos que nunca desarrollaron síntomas) ${ }^{46}$. Además los niños se contagiarían más con las nuevas cepas virales ${ }^{3}$. La ventilación sería la principal herramienta para la prevención de la transmisión viral en el ámbito de las escuelas, y ésta debe ser continua (no alcanza con abrir ventanas antes o después de que lleguen las personas), cruzada (es mejor abrir varias ventanas un poco para facilitar la circulación del aire que abrir mucho una sola), distribuida (para crear una circulación de aire) y medida (a partir de la medición del $\mathrm{CO}_{2}$, que indica la pauta para saber si se está ventilando bien o mal). Los detectores de $\mathrm{CO}_{2}$, dispositivos prácticos y útiles para esta finalidad, 
varían en su costo: entre 60 y 100 dólares o mucho menos los de fabricación local. La recomendación es mantener el $\mathrm{CO} 2$ por debajo de 700 partes por millón ${ }^{45}$. Por encima de esa medida se debe ventilar más, mientras que si los valores están por debajo y hace frío, pueden cerrarse unos centímetros las ventanas por un momento. Es previsible que contar con uno de estos dispositivos por escuela sería costo-efectivo, y mantendrían su utilidad incluso post-pandemia para otras enfermedades de transmisión aérea.

Existe bastante consenso internacional en la suspensión de la presencialidad ante situaciones de muy alta circulación viral, pero más allá de "presencialidad sí" o "presencialidad no", la clave es "presencialidad cómo".

Sin intervenciones de mitigación adicionales, la reapertura de los establecimientos educativos de todos los niveles es probable que aumente la transmisión, esta vez con variantes más infecciosas y posiblemente más virulentas, que resultarán en más bloqueos, más cierres de escuelas y más ausentismo por parte de alumnos y docentes. Aplicando adecuadamente medidas de mitigación comunes y de bajo costo se puede reducir este riesgo. Estas medidas, junto con la vigilancia y la evaluación continuas de la evidencia emergente (que es sumamente dinámica), promoverán tanto la protección de la salud de toda la sociedad, como los logros educativos de los estudiantes, atendiendo a su bienestar y al de sus familias.

Conflictos de interés: Todas las opiniones vertidas en esta editorial son de mi autoría y no se relacionan con las posiciones que podrían tener las instituciones a las que pertenezco. Declaro no tener conflicto de intereses, incluyendo que mis hijos han superado la edad escolar.

Recibido el 24/02/2021 Aceptado el 09/06/2021 y Publicado el 14/06/2021.

\section{Referencias}

1. Castro R, Durán G, Aliaga J, et al.. Informe sobre el Impacto de la presencialidad escolar en los casos confirmados de COVID-19; 2021 . Available from: https://www.ic.fcen.uba.ar/uploads/files/Informe\%20sobre\%20el\%20lmpacto\%20de\%20la\%20presencialidad\%20escolar\%20en\%20los\% 20 casos\%20confirmados\%20de\%20COVID-19\%20.

2. Cochrane Effective Practice and Organisation of Care (EPOC) group. Suggested risk of bias criteria for EPOC reviews; 2017 . Available from: https:// epoc.cochrane.org/sites/epoc.cochrane.org/files/public/uploads/Resources-for-authors2017/suggested risk of bias criteria for epoc reviews.pdf.

3. Centers for Disease Control and Prevention. About Variants of the Virus that Causes COVID-19; 2021. coronavirus/2019-ncov/variants/variant.html [Last access: 2021-06-11]

4. Rubinstein A. Presencialidad escolar: ciencia, pseudociencia y militancia; 2021. Available from: https://www.lanacion.com.ar/sociedad/ presencialidad-escolar-ciencia-pseudociencia-y-militancia-nid16052021/ [Last access: 2021-06-11]

5. Guyatt G, Oxman AD, AkI EA, et al. GRADE guidelines: 1. Introduction-GRADE evidence profiles and summary of findings tables. J Clin Epidemiol. 2011;64(4):383-394. Available from: 10.1016/j.jclinepi.2010.04.026;https://dx.doi.org/10.1016/j.jclinepi.2010.04.026.

6. Schünemann $\mathrm{H}$, zek JB, Guyatt $\mathrm{G}$, et al. Handbook for grading the quality of evidence and the strength of recommendations using the GRADE approach. 2013;Available from: https://gdt.gradepro.org/app/handbook/handbook.html\#: :text=GRADE\%20handbook\&text=The\%20GRADE\% 20handbook\%20describes\%20the, www.gradeworkinggroup.org).

7. Harder T, Sin A, Bosch-Capblanch XM, et al. Towards a framework for evaluating and grading evidence in public health. Health Policy 2015;119(6):732-736. Available from: 10.1016/j.healthpol.2015.02.010.

8. Schunemann HJ, Cuello C, AkI EA, et al. GRADE guidelines: 18. How ROBINS-I and other tools to assess risk of bias in nonrandomized studies should be used to rate the certainty of a body of evidence. J Clin Epidemiol. 2019;111:105-114. Available from: 10.1016/j.jclinepi.2018.01.012.

9. Interrupción y respuesta educativa; 2020. Available from: https://es.unesco.org/covid19/educationresponse [Last access: 2021-06-11].

10. Viner RM, Russell SJ, Croker H, et al. School closure and management practices during coronavirus outbreaks including COVID-19: a rapid systematic review. Lancet Child Adolesc Health. 2020;4(5):397-404. Available from: 10.1016/s2352-4642(20)30095-x;https://dx.doi.org/10.1016/ s2352-4642(20)30095-x

11. Golberstein E, Wen H, Miller BF. Coronavirus Disease 2019 (COVID-19) and Mental Health for Children and Adolescents. JAMA Pediatrics. 2020;174(9):819-819. Available from: 10.1001/jamapediatrics.2020.1456;https://dx.doi.org/10.1001/jamapediatrics.2020.1456.

12. El Gobierno envió al Congreso el proyecto de ley de emergencia Covid; 2021. Available from: https://www.pagina12.com.ar/340933-el-gobiernoenvio-al-congreso-el-proyecto-de-ley-de-emergenc.

13. Rada G, Perez D, Araya-Quintanilla F, et al. Epistemonikos: a comprehensive database of systematic reviews for health decision-making. BMC Med Res Methodol. 2020;20(1):286-286. Available from: 10.1186/s12874-020-01157-x

14. Worby CJ, Chaves SS, Wallinga J, et al. On the relative role of different age groups in influenza epidemics. Epidemics. 2015;13:10-16. Available from: 10.1016/j.epidem.2015.04.003;https://dx.doi.org/10.1016/j.epidem.2015.04.003.

15. Bin-Nafisah S, Alamery AH, Al-Nafesa A, et al. School closure during novel influenza: A systematic review. J Infect Public Health. 2018;11(5):657661. Available from: 10.1016/j.jiph.2018.01.003;https://dx.doi.org/10.1016/j.jiph.2018.01.003.

16. Rashid H, Ridda I, King C, et al. Evidence compendium and advice on social distancing and other related measures for response to an influenza pandemic. Paediatr Respir Rev. 2015;16(2):119-126. Available from: 10.1016/j.prrv.2014.01.003;https://dx.doi.org/10.1016/j.prrv.2014.01.003.

17. Brauner JM, Mindermann S, Sharma M, et al. Inferring the effectiveness of government interventions against COVID-19. Science. 2021;371(6531):eabd9338. Available from: 10.1126/science.abd9338.

18. Cauchemez S, Ferguson NM, Wachtel C, et al. Closure of schools during an influenza pandemic. Lancet Infect Dis. 2009;9(8):473-481. Available from: 10.1016/s1473-3099(09)70176-8;https://dx.doi.org/10.1016/s1473-3099(09)70176-8.

19. Xu W, Li X, Dozier M, et al. What is the evidence for transmission of COVID-19 by children in schools? A living systematic review. J Glob Health. 2020;10(2):21104-21104. Available from: 10.7189/jogh.10.021104.

20. Walsh S, Chowdhury A, Russell S, et al. Do school closures and school reopenings affect community transmission of COVID-19? A systematic review of observational studies. medRxiv. 2021;Available from: 10.1101/2021.01.02.21249146.

21. Suk JE, Vardavas C, Nikitara K, et al. The role of children in the transmission chain of SARS-CoV-2: a systematic review and update of current evidence. MedRxiv. 2020;Available from: 10.1101/2020.11.06.20227264.

22. Muhammed ZF. Effects of school closure on transmission of COVID-19. A rapid systematic review. Kurdistan Journal of Applied Research. 2020;5(3):106-111. Available from: 10.24017/covid.12;https://dx.doi.org/10.24017/covid.12.

23. Yigezu A, Yitayal M, Mirkuzie A, et al. An economic evaluation of Influenza and COVID -19 pandemic prevention and control interventions: a systematic review. Research Square. 2020;Available from: 10.21203/rs.3.rs-121849/v1.

24. Haug N, Geyrhofer L, Londei A, et al. Ranking the effectiveness of worldwide COVID-19 government interventions. Nat Hum Behav. 2020;4(12):13031312. Available from: 10.1038/s41562-020-01009-0;https://dx.doi.org/10.1038/s41562-020-01009-0.

25. Liu Y, Morgenstern C, Kelly J, et al. The impact of non-pharmaceutical interventions on SARS-CoV-2 transmission across 130 countries and territories. BMC Medicine. 2021;19(1):40-40. Available from: 10.1186/s12916-020-01872-8;https://dx.doi.org/10.1186/s12916-020-01872-8

26. Brauner JM, Mindermann S, Sharma M. Inferring the effectiveness of government interventions against COVID-19. Science. 2021;(6531):371-371. Available from: 10.1126/science.abd9338. 
27. Hyde Z. COVID-19, children and schools: overlooked and at risk. Med J Aust. 2021;214(4):190-190. Available from: 10.5694/mja2.50934;https: //dx.doi.org/10.5694/mja2.50934.

28. Hyde Z. Difference in SARS-CoV-2 attack rate between children and adults may reflect bias. Clin Infect Dis. 2021 ;

29. Gurdasani D, Alwan NA, Greenhalgh T, et al. School reopening without robust COVID-19 mitigation risks accelerating the pandemic. The Lancet. 2021;397(10280):1177-1178. Available from: 10.1016/s0140-6736(21)00622-x;https://dx.doi.org/10.1016/s0140-6736(21)00622-x.

30. Lessler J, Grabowski MK, Grantz KH, et al. Household COVID-19 risk and in-person schooling. Science. 2021;372(6546):1092-1097. Available from: 10.1126/science.abh2939; https://dx.doi.org/10.1126/science.abh2939.

31. Brooks SK, Smith LE, Webster RK, et al. The impact of unplanned school closure on children's social contact: rapid evidence review. Euro Surveill. 2020;25(13). Available from: 10.2807/1560-7917.es.2020.25.13.2000188;https://dx.doi.org/10.2807/1560-7917.es.2020.25.13.2000188.

32. Saha S, Saha S. Epidemiological burden of parents being the index cases of COVID-19 infected children. World J Methodol. 2021;11(1):1-14. Available from: 10.5662/wjm.v11.i1.1.

33. Gayatri M. The Implementation of Early Childhood Education in the Time of Covid-19 Pandemic: A Systematic Review. Humanities \& Social Sciences Reviews. 2020;8(6):46-54. Available from: 10.18510/hssr.2020.866.

34. Krishnaratne S, Pfadenhauer LM, Coenen M, et al. Measures implemented in the school setting to contain the COVID-19 pandemic: a rapid scoping review. Cochrane Database Syst Rev. 2020;12:13812-13812. Available from: 10.1002/14651858.cd013812;https://dx.doi.org/10.1002/14651858. cd013812.

35. Lo-Moro G, Sinigaglia T, Bert F, et al. Reopening Schools during the COVID-19 Pandemic: Overview and Rapid Systematic Review of Guidelines and Recommendations on Preventive Measures and the Management of Cases. Int J Environ Res Public Health. 2020;17(23):8839-8839. Available from: 10.3390/ijerph17238839;https://dx.doi.org/10.3390/ijerph17238839.

36. Pan American Health Organization. Considerations for School-Related Public Health Measures for Populations in Vulnerable Conditions in the Context of COVID-19; 2021. Available from: https://iris.paho.org/handle/10665.2/53317 [Last access: 2021-06-14].

37. World Health Organization. Checklist to support schools re-opening and preparation for COVID-19 resurgences or similar public health crises; 2020. Available from: https://www.who.int/publications/i/item/9789240017467 [Last access: 2021-06-14].

38. Argentina Ministerio de Educación Consejo Federal de Educación. Protocolo marco y lineamientos federales para el retorno a clases presenciales en la educación obligatoria y en los institutos superiores; 2020. Available from: https://evidencia.typeset.io/edit/5b003aa1-6c14-4c0c-aa48193e34baf0a4\# [Last access: 2021-06-14].

39. Argentina Ministerio de Salud. Plan estratégico para la vacunación contra la COVID-19 en la República Argentina; 2020. Available from: https: //www.argentina.gob.ar/sites/default/files/coronavirus-vacuna-plan-estrategico-vacunacion-covid-19-diciembre-2020.pdf [Last access: 2021-06-14].

40. Kreuter F, Barkay N, Bilinski A et al. Partnering with a global platform to inform research and public policy making. What needs to be in place to make a global COVID-19 survey work? Surv Res Methods. 2020;14(2):159-163. Available from: 10.18148/srm/2020.v14i2.7761.

41. Lessler J, Grabowski MK, Grantz KH, et al. Household COVID-19 risk and in-person schooling. Science. 2021;372(6546):1092-1097. Available from: 10.1126/science.abh2939.

42. Zimmerman KO, Akinboyo IC, Brookhart MA, et al. Incidence and Secondary Transmission of SARS-CoV-2 Infections in Schools. Pediatrics. 2021;147(4):e2020048090. Available from: 10.1542/peds.2020-048090

43. Argentina Ministerio de Educación. Los casos positivos de COVID en las escuelas representan el $0.12 \%$ en estudiantes matriculados; 2021. Available from: https://www.argentina.gob.ar/noticias/los-casos-positivos-de-covid-en-las-escuelas-representan-el-012-en-estudiantesmatriculados [Last access: 2021-06-14].

44. Germany The Federal Government. Infection Protection Act in the German Bundestag. Nationwide emergency brake passed; 2021 . Available from: https://www.bundesregierung.de/breg-en/search/nationwide-emergency-brake-1889136 [Last access: 2021-06-14].

45. Greenhalgh T, Jimenez JL, Prather KA, et al. Ten scientific reasons in support of airborne transmission of SARS-CoV-2. Lancet. 2021;397(10285):1603-1605. Available from: 10.1016/s0140-6736(21)00869-2;https://dx.doi.org/10.1016/s0140-6736(21)00869-2.

46. Johansson MA, Quandelacy TM, Kada S, et al. SARS-CoV-2 Transmission From People Without COVID-19 Symptoms. JAMA Network Open. 2021;4(1):e2035057-e2035057. Available from: 10.1001/jamanetworkopen.2020.35057; https://dx.doi.org/10.1001/jamanetworkopen.2020.35057. 